\title{
Quantifying the Robustness of Topological Slow Light
}

\author{
Guillermo Arregui $\odot,{ }^{1}$ Jordi Gomis-Bresco $\odot,{ }^{1, *}$ Clivia M. Sotomayor-Torres $\odot,{ }^{1,2}$ and Pedro David Garcia $\odot^{1, \dagger}$ \\ ${ }^{1}$ Catalan Institute of Nanoscience and Nanotechnology (ICN2), CSIC and BIST, Campus UAB, Bellaterra, 08193 Barcelona, Spain \\ ${ }^{2}$ ICREA-Institució Catalana de Recerca i Estudis Avançats, 08010 Barcelona, Spain
}

(Received 31 July 2020; accepted 15 December 2020; published 14 January 2021)

\begin{abstract}
The backscattering mean free path $\xi$, the average ballistic propagation length along a waveguide, quantifies the resistance of slow light against unwanted imperfections in the critical dimensions of the nanostructure. This figure of merit determines the crossover between acceptable slow-light transmission affected by minimal scattering losses and a strong backscattering-induced destructive interference when the waveguide length $L$ exceeds $\xi$. Here, we calculate the backscattering mean free path for a topological photonic waveguide for a specific and determined amount of disorder and, equally relevant, for a fixed value of the group index $n_{g}$ which is the slowdown factor of the group velocity with respect to the speed of light in vacuum. These two figures of merit, $\xi$ and $n_{g}$, should be taken into account when quantifying the robustness of topological and conventional (nontopological) slow-light transport at the nanoscale. Otherwise, any claim on a better performance of topological guided light over a conventional one is not justified.
\end{abstract}

DOI: $10.1103 /$ PhysRevLett.126.027403

Slowing the speed of a light pulse down to human pace (meters per second) requires complex interference effects [1] which manifest as a flat dispersion relation $\nu=\nu(\mathbf{k})$, where $\mathbf{k}$ is the conserved wave vector and $\nu(\mathbf{k})$ the frequency. The group velocity $v_{g}$ of this slow light is determined by the derivative of the flat band and the slowdown factor is given by the group index as $n_{g}=c / v_{g}$, where $c$ is the speed of light in vacuum. $n_{g}$ is the figure of merit for slow light and it determines the enhancement factor for diverse applications such as optical nonlinearities [2], optical switching [3], pulse delay [4], quantum optics [5], optical storage [6], and optical gain [7]. A strategy to bring slow light to the nanoscale exploits optical resonances built up by nanostructuring a dielectric or semiconductor material with low absorption, such as silicon at telecom wavelengths. Flat bands arise naturally in these systems based on the periodic modulation of the refractive index at optical or near infrared wavelengths $[8,9]$ for which the group index diverges as $n_{g} \propto(\partial \nu / \partial k)^{-1}$ in the ideal situation. However, in real devices there is a limitation to the maximum $n_{g}$ achievable due to slight deviations of the fabricated parameters compared to the designed values. Even fluctuations in the nanometer range [10] give rise to backscattering of the guided light, inducing a strong interference [11], a photonic manifestation of Anderson localization in low dimensions [12]. Imperfection limits slow light in conventional photonic waveguides to maximum values around $n_{g} \approx 100$ for very short waveguides with lengths $L \simeq 5 \mu \mathrm{m}$, much lower than the $n_{g}$ values observed in atomic systems [1] but still sufficiently large to explore weak light-matter interaction leading to cavityquantum electrodynamic phenomena [5]. In this Letter, we confirm that this limitation may be overcome by exploiting photonic topological effects that purely arise from engineering the lattice geometry.

Topological photonics has emerged very recently as a competitive approach for robust light transport [13], something extremely appealing for technological applications. In time-invariant topological insulators based on the quantumspin Hall effect [14] and the valley-Hall effect [15], topology emanates from the breaking of particular spatial symmetries. In such implementations, reciprocity imposes the existence of the counterpropagating mode at $-\mathbf{k}$. This time-reversed edge state carries the opposite value of a binary degree of freedom that plays the role of a pseudospin [14]. In this case, the key open question is whether or not backscattering is reduced and the answer will depend on whether or not the existing structural disorder preserves the pseudospin value. Recent ground breaking experiments reported robustness in terms of a certain lack of structural back reflection when precisely shaped local defects were introduced in different topological waveguides [16]. However, this claimed robustness still needs to be systematically quantified and compared with state-of-the art conventional (nontopological) ones. Here, we engineer slow light in a valley-Hall waveguide [15] to calculate its backscattering length $\xi$ versus disorder and $n_{g}$ and we compare the results to those of a conventional photonic waveguide.

For our analysis, we focus on the parity-symmetry breaking valley-Hall approach [15]. When applied to photonic slabs, the topological edge states at an interface between valley-Hall crystals of opposite $K$-valley pseudospin lay below the light line of the slab and are decoupled 


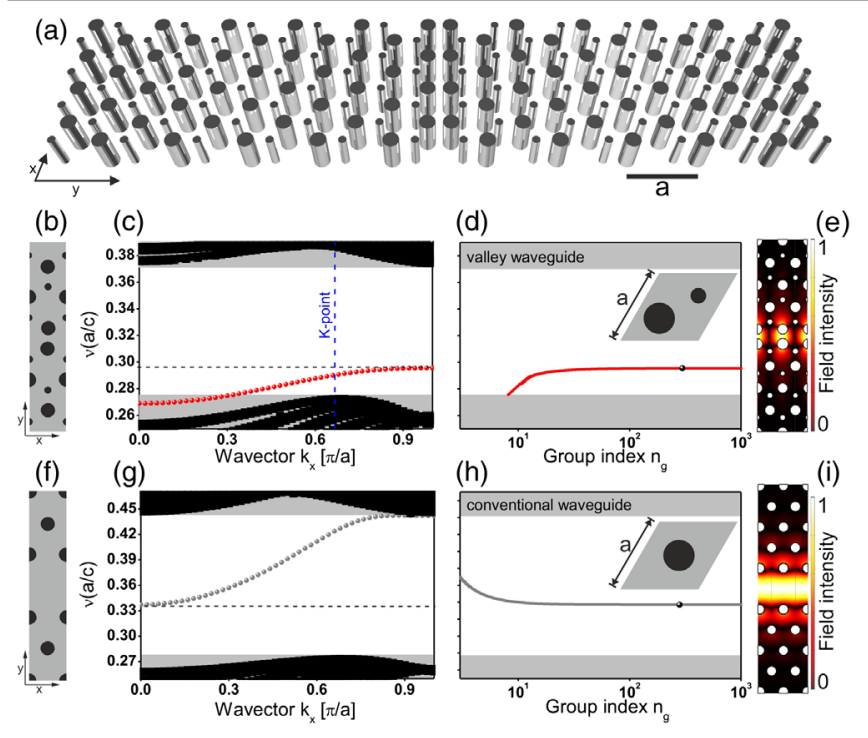

FIG. 1. Slow light in topological waveguides. (a) Valley topological waveguide (illustration) formed at the interface of two valley crystals with different topological invariants as proposed in Ref. [15]. (b) Dielectric function distribution (black corresponds to silicon and gray to air). (c) Dispersion relation $\nu=\nu(k)$, and (d) calculated group index of the interface topological edge state (inset displays the unit cell). For reference, we design a conventional photonic waveguide obtained by leaving a row of pillars from a triangular lattice (f) with a dispersion relation and a group index plotted in $(\mathrm{g})$ and $(\mathrm{h})$, respectively. The black point in the group index curves denotes the frequency at which each waveguide has a $n_{g} \approx 300$. The electromagnetic field intensities calculated at these frequencies and for perfect waveguides are plotted in (e) and (i).

from the radiation continuum. In principle, these edge states have no intrinsic out-of plane losses and only fabrication imperfection can induce coupling to radiating modes. Even in that situation, in-plane backscattering is largely the dominant loss mechanism at large values of $n_{g}$ [17] so it is enough to consider the system as two dimensional to capture the physics of slow-light backscattering, something not possible with other implementations of topological photonics [18]. Here, we set up twodimensional simulations instead of the more computationally expensive three-dimensional slab. Figure 1(a) displays an illustration of a section of a fully two-dimensional valley-Hall waveguide formed at the interface of two valley crystals. The valley crystals are created with a triangular lattice where the unit cell is formed by two circular silicon pillars surrounded by air with different diameters $d_{1}=0.4 a$ and $d_{2}=0.2 a$, where $a$ is the lattice constant with dielectric constant $\varepsilon_{S i}=12$ and $\varepsilon_{\text {air }}=1$, respectively. The two-dimensional layout of the waveguide is plotted in Fig. 1(b). For $d_{1}=d_{2}$, the system preserves the $C_{6 v}$ symmetry and supports a symmetry-protected gapless band structure between the first and second lowest energy bands for transverse magnetic polarized light. For different pillar diameters, the spatial inversion symmetry is broken thus opening a band gap between these two bands [15] (see Supplemental Material [19]). Figure 1(c) shows the calculated dispersion relation of the waveguide and in Fig. 1(d) we plot the group index of the topological edge mode with vanishing $v_{g}$ at the cutoff frequency $\nu=0.2955(c / a)$, corresponding to $\nu=177 \mathrm{THz}$ for $a=500 \mathrm{~nm}$. For reference, we use a standard photonic crystal waveguide obtained by leaving out a row of pillars in a triangular lattice of silicon pillars surrounded by air with a pillar diameter $d=0.4 a$ and the same lattice unit as the topological waveguide, as shown in Fig. 1(f). The conventional guided mode presents an ideally vanishing group velocity when approaching the cutoff frequency of the waveguide at $\nu=0.3372(c / a)$, as shown in Figs. 1(g) and 1(h). Here, the cutoff of the topological and conventional waveguide lie on the $X$ and $\Gamma$ point, respectively. The ideal spatial field-intensity distributions in both waveguides and at frequencies corresponding to $n_{g}=300$ in both cases are plotted in Figs. 1(e) and 1(i) for reference, which shows a similar level of light confinement. Using silicon pillars surrounded by air instead of the usual air holes in silicon enables us to flatten the dispersion relation of both topological and conventional guided modes successfully without the need of local perturbations as in Ref. [22] or progressive interfaces as done in Ref. [23]. The parameters to obtain flat bands in the valley-Hall waveguide are detailed in the Supplemental Material [19].

The backscattering length $\xi$ is the average ballistic propagation distance along the waveguide in the absence of any other major loss mechanism [17]. A slow-light waveguide becomes virtually useless when $L \gg \xi$. Interestingly, $\xi$ is linked to the density of optical states of the waveguide, $\rho$, at least for a weak perturbation [17,24], as $\xi \propto \rho^{-2}=(\partial \nu / \partial k)^{2}$. Intuitively, a larger density of optical states induces a larger probability of scattering, thus reducing the value of $\xi$. As $n_{g} \propto$ $(\partial \nu / \partial k)^{-1}=\rho$, both the group index and the backscattering length are intrinsically linked to each other via the $\rho$, at least in conventional photonic crystal waveguides [25]. Despite substantial theoretical work on $\xi$ in nontopological electronic [26-28] and photonic transport [17,25,29], this parameter has only been explored recently in topological waveguides [30] although ignoring $n_{g}$. Besides this, only intrinsic out-of plane losses of topological guided modes in nondisordered photonic crystal slabs have been analyzed [31]. We calculate $\xi$ in perturbed topological and conventional photonic crystal waveguides as

$$
-\frac{x}{\xi(\nu)}=\langle\ln [I(\nu)]\rangle
$$

where $I$ is the finite-element solution of the electromagnetic field intensity emitted by a dipole at frequency $\nu, x$ is the distance from the dipole position along the waveguide and the brackets indicate the statistical ensemble average over different configurations of positional disorder. In Eq. (1), 
the field intensity $I$ is normalized by its (maximum) value at the source position which is the same in each calculation. Our simulation domain has a length $L=200 a$ in the $x$ direction, eleven unit cells on each side of the waveguide in the $y$ direction and it is all surrounded by perfectly matched layers to mimic an open system. In some cases, when the calculated value of $\xi$ exceeds $L=200 a$, we extend the simulation domain to $L=500 a$ which also allowed us to double-check some of the obtained results. To simulate the effect of fabrication imperfection, we randomize the position of the pillars around their ideal value according to a normal distribution which standard deviation $\sigma$ is our measure of disorder (more details in the Supplemental Material [19]). It is important to remark the fact that the backscattering length is a universal parameter in onedimensional transport [32,33] and any mesoscopictransport observable in these systems depends only on $\xi$, regardless of the microscopic details of the medium considered, i.e., regardless of it being silicon pillars surrounded by air or air inclusions in silicon.

The electromagnetic field intensity excited by a dipole source oscillating at the leftmost edge of the waveguide is shown in Fig. 2. The oscillation frequency is chosen such that both the topological (left) and the conventional (right) waveguide would enable slow light transmission with $n_{g}=300$ in the absence of any imperfection. The figure shows calculations corresponding to varying configurations of positional disorder for which the positions of the pillars are randomized with a fixed $\sigma=0.001 a$. As shown in Fig. 2(a), the excited Bloch modes in the topological waveguide are just slightly perturbed, which reveals a rather weak backscattering for this large group index value. However, strong backscattering interference prevents light transport in the conventional waveguide, as revealed by the different examples plotted in Fig. 2(b). As $\xi$ is a statistical parameter, this requires an ensembleaverage calculation of many (ideally all) different disorder configurations. In numerical experiments, as the one performed here, $\xi$ is easily obtained by computing the position-dependent field excited by the dipole source in the conditions described above for several structural configurations with the same nominal amount and type of disorder. Figures 2(c) and 2(d) show the intensity pattern after ensemble averaging the electromagnetic field excited by this emitter over twenty different realizations, the envelope of which decays exponentially from the position of the source with a sufficiently well-averaged slope, as plotted along the waveguide axis in Figs. 2(e) and 2(f) for $n_{g}=300$.

The calculated $\xi$ vs $n_{g}$ is plotted in Fig. 3(a) for the topological (solid-red circles) and conventional waveguide (open-black circles). $\xi$ is calculated for a fixed amount of positional disorder $\sigma=0.001 a$, which corresponds to fluctuations of $\approx 0.5 \mathrm{~nm}$ for $a=500 \mathrm{~nm}$. This is a realistic measure of the residual imperfection resulting from a stateof-the art fabrication process [10,34]. At such a disorder level, the topological waveguide is more robust than standard conventional slow-light waveguides when the full phase is randomized, thus mimicking the effect of imperfection in real systems. Topological waveguides suffer much less backscattering than conventional ones [see Fig. 3(a)] with a $\xi$ comparable to the waveguide length for large group index values. Even at large values of the group index, $n_{g} \simeq 1000$, the interface edge mode of the
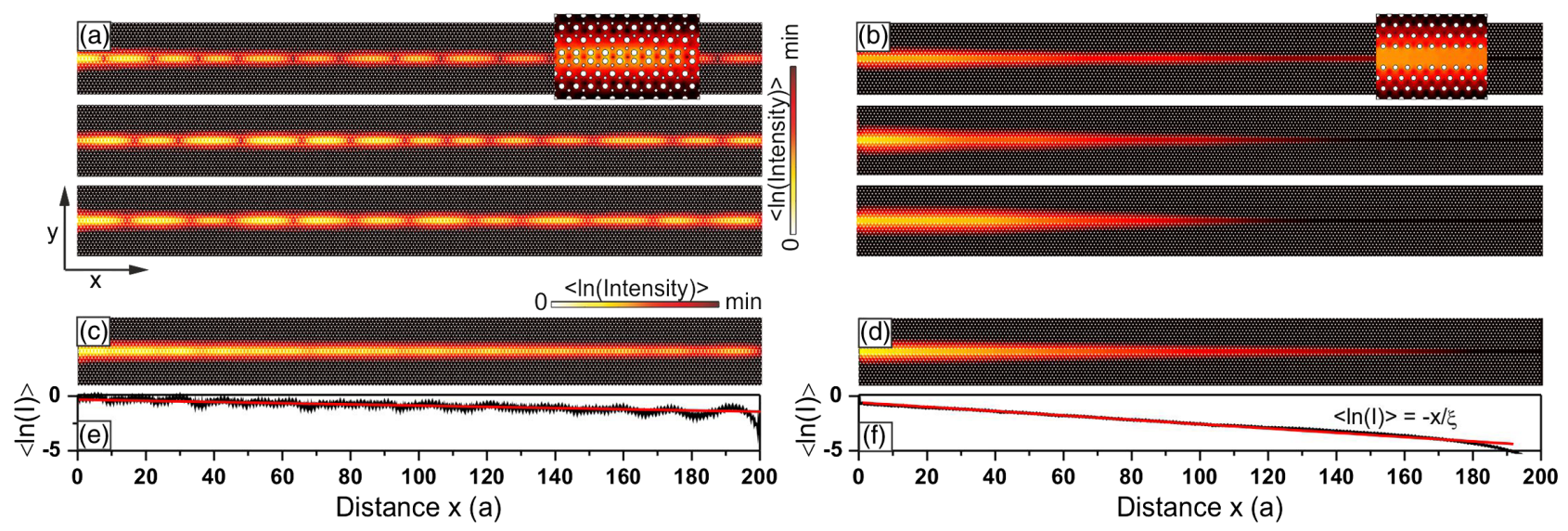

FIG. 2. Backscattering in topological slow-light waveguides. (a) Normalized electromagnetic-field intensity excited by a dipole emitter positioned on the left side of a disordered valley-Hall waveguide at a frequency $\nu=0.2955(c / a)$, where $a$ is the lattice constant and $c$ is the speed of light in vacuum. Three different configurations of randomized positions of the pillars are plotted with a standard deviation of $\sigma=0.001 a$. (b) Calculations for a conventional photonic waveguide where the dipole emitter is excited at a frequency $\nu=0.3372(c / a)$. In (a) and (b), the dipole emission frequency corresponds to a group index of $n_{g}=300$. (c) and (d) show the ensemble-averaged electromagnetic field intensity excited by the dipole source at the same frequencies of (a) and (b). For this calculation, over twenty configurations of positional disorder of the pillars with $\sigma=0.001 a$ were averaged. (e) and (f) illustrate the calculated ensemble-averaged electromagnetic field-intensity profile along the topological and conventional waveguide axis, respectively. The localization length is extracted from the inverse of the exponential decay slope. 

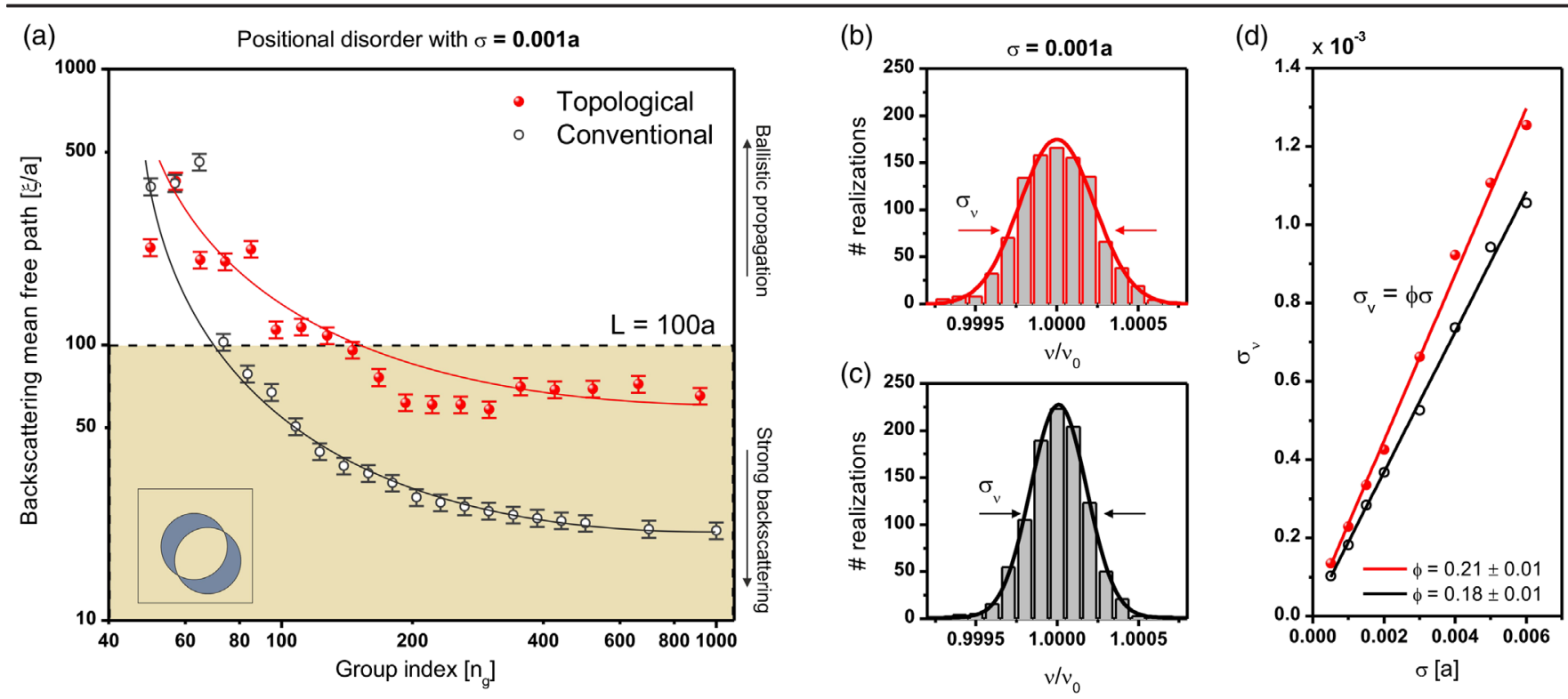

FIG. 3. Backscattering length versus group index in topological waveguides. (a) $\xi$ vs $n_{g}$ calculated in a valley (solid-red circles) and a conventional photonic crystal waveguide (open-black circles) for a fixed amount of disorder in the position of the pillars $(\sigma=0.001 a$ ). The shaded area indicates a waveguide of length $L=100 a$ and the lines are guides to the eye. (b) and (c) Distributions of the cutoff frequency calculated in a topological and a conventional waveguide for $10^{4}$ different configurations of positional disorder with $\sigma=0.001 a$. The calculated frequency is formalized to the frequency of the unperturbed structures $\nu_{0}$. (d) Standard deviation of the distribution of the frequency shifts $\pm \Delta \nu$ as calculated in (b) and (c) vs the strength of the perturbation. From the relation $\sigma_{v}=\phi \sigma$, we quantify the effective impact of disorder in the waveguides with the parameter $\phi$.

topological waveguide is slightly perturbed when compared to the strong backscattering suffered by the conventional one, as shown in the different configurations plotted in Fig. 2(a). Here, it is relevant to discard mechanisms that could affect in a different manner both systems and yield the data plotted in Fig. 3(a), rather than the topological protection. Small geometrical perturbations in the pillars of the waveguides result in positive or negative energy shifts $\pm \triangle \nu$ of the dispersion relation of a guided mode [35], where $\Delta \nu \ll \nu_{0}$. Here, the $\nu_{0}$ is the unperturbed guidedmode cutoff frequency, i.e., the frequency for which the group index is maximum. To quantify this effect, we calculate the cutoff frequency of the guided mode in both systems after perturbing the waveguides by introducing a fixed positional disorder $\sigma=0.001 a$. Figures 3(b) and 3(c) plot the distributions of this cutoff frequency, $\nu$, for $10^{4}$ different configurations normalized by $\nu_{0}$. For the same amount of positional disorder the impact, in terms of the standard deviation of the distribution $\sigma_{\nu}$, is stronger on the topological edge state than on the conventional guided mode, as $\sigma_{\nu}$ is clearly narrower for the former. We evaluate the dependence of $\sigma_{v}=\phi \sigma$ on positional disorder strength for a wide range of values in both waveguides, where the parameter $\phi$ quantifies the effective impact of disorder in both cases. As plotted in Fig. 3(d), the topological waveguide suffers a larger effective impact of disorder than the conventional waveguide, i.e., disorder shifts the energy of its unperturbed cutoff frequency in a more pronounced way, which underlines even more the potential of the topological protection observed here at very large values of $n_{g}$, the main result of this Letter.

The calculated $\xi$ vs disorder is plotted in Fig. 4 where the value of the group index is kept fixed at $n_{g}=100$ (a) and $n_{g}=500$ (b). $\xi$ has a different dependence on the disorder strength $\sigma$ on both waveguides. This is clear in Figs. 4(a) and 4(b), where the slope of this dependence is markedly different for the topological and conventional waveguides and becomes even more different when the value of $n_{q}$ increases. To quantify this dependence, we fit the calculated values as $\xi \sim \sigma^{-\beta}$. We plot the dependence of the exponent $\beta$ in Fig. 4(c) on $n_{g}$. This dependence has been studied in terms of the Lyapunov exponent $\gamma$, which under certain hypotheses fulfilled here quantifies the exponential decay of the eigenfunctions in a disordered system [36]. $\gamma$ is the inverse of $\xi$, the localization length. The dependence of $\gamma$ on the amount of disorder $\gamma \sim \sigma^{\beta}$ has been analyzed in perturbed periodic photonic structures [37] where a value of $\beta \sim 2$ is obtained in the bulk of the dispersion relation. Here, we recover this dependence in both waveguides for low values of $n_{g}$. Near the cutoff frequency, the parameter $\beta$ is expected to converge to $2 / 3$ as observed in Refs. $[37,38]$. Here, $\beta$ displays a very different behavior in both waveguides close to the cutoff frequency. While $\beta \sim 2 / 3$ for the conventional waveguide [37], $\beta \sim 3 / 2$ for the topological one. We also extract a critical level of disorder $\sigma_{c}$, above which light in the conventional waveguide backscatters less than the topological one. $\sigma_{c}$ is obtained at the crossing between the 


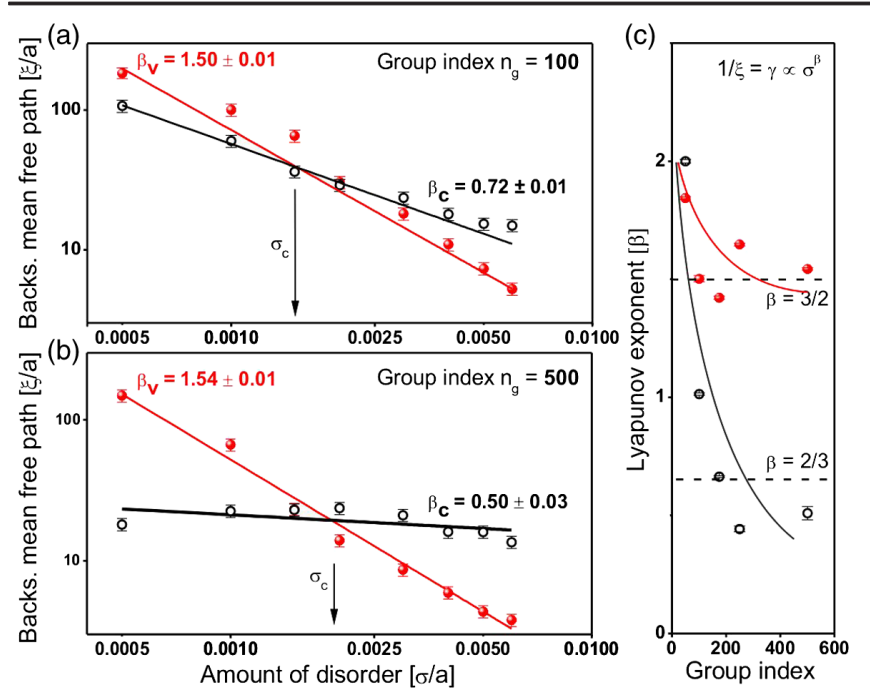

FIG. 4. Backscattering length versus disorder in topological waveguides. Calculated backscattering length vs positional pillar disorder for a fixed group index value $n_{g}=100$ (a) and $n_{g}=500$ (b), in solid-red and open-black circles for the topological and the conventional waveguide, respectively. The dependence of the backscattering length vs the strength of disorder is fitted as $\xi \sim \sigma^{-\beta}$. Above a critical level of disorder $\sigma_{c}$ the conventional waveguide sufferers less backscattering than the topological one. $\sigma_{c}$ is estimated from the crossing of the fits. (c) The fitting parameter $\beta$ plotted for a wide range of values of the group index.

fits of $\xi$ for both waveguides. Above $\sigma_{c}$, the probability of a pseudospin flip upon scattering of the Bloch mode increases, leading to a decreasing $\xi$.

In conclusion, one of the key elements to quantify the robustness of photonic waveguides is to randomize the full phase to mimic real imperfection due to the fabrication process, the real limitation in slow-light transport. Calculating the backscattering length linked to the group index enables us to quantify the robustness of a topological edge state, as these two parameters are related to each other through the density of optical states. Current proposals of topological photonic phases as the valleyHall effect are quantitatively, by almost five times, more robust than standard conventional waveguides with small disorder levels, although this protection is lost at higher imperfection amount. The analysis carried out here is based on a particular system of silicon pillars surrounded by air but the approach is completely generic and can be implemented for any other single-mode waveguide with arbitrary design. The large group index values calculated here, $n_{g} \simeq 1000$, provide a promising platform for highly efficient strong light-matter interaction [39] where photon transmission over hundreds of microns is relatively backscattering free. Future work to evaluate topological invariants [40] of different topological implementations will provide additional insight into the relationship between the backscattering length evolution with disorder level and the protection granted by topology.
This work was supported by the European Commission H2020 FET Proactive project TOCHA (No. 824140). The authors acknowledge funding by the Severo Ochoa program from Spanish MINECO (Grant No. SEV-2019-0706) and the Plan Nacional RTI2018-093921-A-C44 (SMOOTH) and CERCA Programme/Generalitat de Catalunya. G. A. was supported by a H2020 MSC cofund action, a BIST Ph.D. fellowship, and P.D. G. by a Ramon y Cajal Fellowship No. RyC-2015-18124.

*jordi.gomis@icn2.cat david.garcia@icn2.cat

[1] L. V. Hau, S. E. Harris, Z. Dutton, and C. H. Behroozi, Nature (London) 397, 594 (1999).

[2] C. Monat, B. Corcoran, M. Ebnali-Heidari, C. Grillet, B. J. Eggleton, T. P. White, L. O'Faolain, and T. F. Krauss, Opt. Express 17, 2944 (2009).

[3] M. Bajcsy, S. Hofferberth, V. Balic, T. Peyronel, M. Hafezi, A. S. Zibrov, V. Vuletic, and M. D. Lukin, Phys. Rev. Lett. 102, 203902 (2009).

[4] M. D. Stenner, M. A. Neifeld, Z. Zhu, A. M. C. Dawes, and D. J. Gauthier, Opt. Express 13, 9995 (2005).

[5] M. Arcari et al., Phys. Rev. Lett. 113, 093603 (2014).

[6] C. Sayrin, C. Clausen, B. Albrecht, P. Schneeweiss, and A. Rauschenbeutel, Optica 2, 353 (2015).

[7] S. Ek, P. Lunnemann, Y. Chen, E. Semenova, K. Yvind, and J. Mork, Nat. Commun. 5, 5039 (2014).

[8] T.F. Krauss, , R. M. De La Rue, and S. BrandNature (London) 383, 699 (1996).

[9] T. Baba, Nat. Photonics 2, 465 (2008).

[10] P. D. García, A. Javadi, H. Thyrrestrup, and P. Lodahl, Appl. Phys. Lett. 102, 031101 (2013).

[11] J. Topolancik, B. Ilic, and F. Vollmer, Phys. Rev. Lett. 99, 253901 (2007).

[12] P. W. Anderson, Phys. Rev. 109, 1492 (1958).

[13] T. Ozawa et al., Rev. Mod. Phys. 91, 015006 (2019).

[14] L. H. Wu and X. Hu, Phys. Rev. Lett. 114, 223901 (2015).

[15] T. Ma and G. Shvets, New J. Phys. 18, 025012 (2016).

[16] X. T. He, E.-T. Liang, J.-J. Yuan, H.-Y. Qiu, X.-D. Chen, F.-L. Zhao, and J.-W. Dong, Nat. Commun. 10, 872 (2019).

[17] S. Mazoyer, J. P. Hugonin, and P. Lalanne, Phys. Rev. Lett. 103, 063903 (2009).

[18] E. Sauer, J. P. Vasco, and S. Hughes, Phys. Rev. Research 2, 043109 (2020).

[19] See Supplemental Material at http://link.aps.org/supplemental/ 10.1103/PhysRevLett.126.027403 for detailed description of the lattice geometry, finite-element calculation details and the convergence of the results, which includes Refs. [14,20,21].

[20] A. Rycerz, J. Tworzydło, and C. Beenakker, Nat. Phys. 3, 172 (2007).

[21] D. Xiao, W. Yao, and Q. Niu, Phys. Rev. Lett. 99, 236809 (2007).

[22] T. Baba and D. Mori, J. Phys. D 40, 2659 (2007).

[23] J. Guglielmon and M. C. Rechtsman, Phys. Rev. Lett. 122, 153904 (2019).

[24] P. D. García, S. Smolka, S. Stobbe, and P. Lodahl, Phys. Rev. B 82, 165103 (2010). 
[25] P. D. García, G. Kiršanskè, A. Javadi, S. Stobbe, and P. Lodahl, Phys. Rev. B 96, 144201 (2017).

[26] A. García-Martín and J. J. Sáenz, Phys. Rev. Lett. 87, 116603 (2001).

[27] L. S. Froufe-Pérez, P. García-Mochales, P. A. Serena, P. A. Mello, and J. J. Sáenz, Phys. Rev. Lett. 89, 246403 (2002).

[28] A. MacKinnon and B. Kramer, Phys. Rev. Lett. 47, 1546 (1981).

[29] V. Savona, Phys. Rev. B 83, 085301 (2011).

[30] B. Orazbayev and R. Fleury, Nanophotonics 8, 8 (2019).

[31] E. Sauer, J. P. Vasco, and S. Hughes, Phys. Rev. Research 2, 043109 (2020).

[32] P. Sheng, Introduction to Wave Scattering, Localization, and Mesoscopic Phenomena (Academic Press, San Diego, 1995).
[33] S. Smolka, H. Thyrrestrup, L. Sapienza, T. B. Lehmann, K. R. Rix, L. S. Froufe-Pérez, P. D García, and P. Lodahl, New J. Phys. 13, 063044 (2011).

[34] Y. Lai, S. Pirotta, G. Urbinati, D. Gerace, M. Minkov, V. Savona, A. Badolato, and M. Galli, Appl. Phys. Lett. 104, 241101 (2014).

[35] R. Faggiani et al., Sci. Rep. 6, 27037 (2016).

[36] R. Carmona, Duke Math. J. 49, 191 (1982).

[37] Y. A. Godin, S. Molchanov, and B. Vainberg, Waves Random Complex Media 21, 135 (2011).

[38] F. M. Izrailev, A. A. Krokhin, and N. M. Makarov, Phys. Rep. 512, 125 (2012).

[39] A. Goban et al., Nat. Commun. 5, 3808 (2014).

[40] M. Blanco de Paz et al., Adv. Quantum Technol. 3, 1900117 (2020). 